\title{
A thermoviscoelastic beam model for brakes
}

Kenneth Kuttler

klkuttle@math.byu.edu

J. Bajkowski

J. R. Fernandez

M. Shillor

Follow this and additional works at: https://scholarsarchive.byu.edu/facpub

Part of the Mathematics Commons

Original Publication Citation

European Journal of Applied Mathematics, 15(24), pp 181-22.

\section{BYU ScholarsArchive Citation}

Kuttler, Kenneth; Bajkowski, J.; Fernandez, J. R.; and Shillor, M., "A thermoviscoelastic beam model for brakes" (2004). Faculty Publications. 459.

https://scholarsarchive.byu.edu/facpub/459

This Peer-Reviewed Article is brought to you for free and open access by BYU ScholarsArchive. It has been accepted for inclusion in Faculty Publications by an authorized administrator of BYU ScholarsArchive. For more information, please contact ellen_amatangelo@byu.edu. 


\title{
A thermoviscoelastic beam model for brakes
}

\author{
J. BAJKOWSKI ${ }^{1}$, J. R. FERNÁNDEZ ${ }^{2}$, \\ K. L. KUTTLER ${ }^{3}$ and M. SHILLOR ${ }^{4}$ \\ ${ }^{1}$ Institute of Machine Design Fundamentals, Warsaw University of Technology, Warsaw, Poland \\ ${ }^{2}$ Departamento de Matemtica Aplicada, Universidade de Santiago de Compostela, 15706 Santiago \\ de Compostela, Spain \\ ${ }^{3}$ Department of Mathematics, Brigham Young University, Provo, UT 84602, USA \\ ${ }^{4}$ Department of Mathematics and Statistics, Oakland University, Rochester, MI 48309, USA
}

(Received 26 February 2002; revised 13 October 2003)

\begin{abstract}
A model for the dynamic thermomechanical behavior of a viscoelastic beam which is in frictional contact with a rigid rotating wheel is presented. It describes a simple braking system in which the wheel comes to a stop as a result of the frictional traction generated by the beam. Friction is modelled with a temperature and slip rate dependent coefficient of friction. Frictional heat generation is taken into account as well as the wheel temperature evolution, and the wear of the beam's contacting end. The model is formulated as a variational inequality. A FEM numerical scheme for the model is described, implemented, and the results of numerical simulations are shown.
\end{abstract}

\section{Introduction}

This work deals with modelling, analysis and numerical simulations of a braking system with simplified geometry. The setting consists of a rigid rotating wheel that comes to a stop as a result of frictional traction generated by contact with a thermoviscoelastic beam. The frictional heat generated during the process is taken into account, and so are the evolution of the wear of the beam's contacting end and the wheel's temperature field. The purpose of this simplified mechanical setting is to allow for the study of the braking process dynamics, while avoiding some of the mathematical difficulties arising in two-or three-dimensions. Although the setting is simplified, the model derived is complex, realistic, and takes into account the main physical phenomena involved in the thermomechanical braking process. Moreover, it is easier to set up experimentally and to perform measurements near the contact point.

The process has important applications in the automotive and aeronautical industries: car, plane or train braking systems, are the more prominent examples. Indeed, the thermomechanical process of frictional contact is an everyday occurrence, but rigorous mathematical investigation of realistic models for the process is rather complicated since the process is strongly nonlinear. Recent progress in nonsmooth mechanics reached a point where such problems can be modelled and the existence of solutions established. Related results, for simplified aspects of the problem can be found in $[6,16,36]$ and 
references therein. Related results and review of current publications can be found in several works $[13,25,30,31]$, and the references therein.

General mathematical models for thermoviscoelastic dynamic frictional contact have been investigated recently $[3,4,8]$, and a comprehensive theory is currently emerging. Quasistatic problems can be found in several works [29, 31, 35]. Related results for various geometries and simple settings in engineering literature can be found $[6,16,36,2,26]$, along with one-dimensional thermoviscoelastic contact problems [11, 12].

This process is of the pin-on-disc type [10,28], which is commonly used for experimental determination of wear. However, our interest lies in the full process of braking, which involves the rotation speed as an unknown, as well as the thermal aspects. A model for a pin-on-disc wear experiment can be obtained from our model by simple modifications, and the results of this work apply to that case too. Modifications of the model for the process where the beam's end is in close proximity to a rotating disc, such as in the case of a reading head of a computer hard drive, are easy to make, but fall outside of the scope of this work.

In this paper, we construct a general model for the process of dynamic contact between a rigid rotating wheel and a thermoviscoelastic beam. It includes: dynamic vibrations of the beam, evolution of its and the wheel's temperature fields, frictional contact and the associated heat generation, slowing down of the wheel's rotational velocity, and the wear of the beam's contacting tip. The model is developed in $\S 2$ and the classical formulation is summarized in (2.8)-(2.24). It consists of a system of linear partial differential equations coupled with an ordinary differential equation and a differential inclusion, and nonlinear boundary conditions. However, we deal with a contact problem which is non-smooth, and so the classical formulation is inadequate, since, in general, there are no classical solutions for such systems. Therefore, we derive a weak formulation for the model in $\S 3$, and then we set it in an abstract form using multivalued operators. The existence of a weak solution for the model has been established in a companion work [23], where a version of the abstract existence theorem in Kuttler \& Shillor [19] has been applied to the problem. We recall this result in $\S 3$ as well. Since we deal with set-valued inclusions, the uniqueness of the solutions remains an open and difficult question. However, the uniqueness of the weak solution for the problem when the wheel temperature and its rotation speed are known was shown in Kuttler et al. [23].

In $\S 4$ an FEM numerical algorithm for the problem is developed, and the simulations resulting from its implementation are presented in $\S 5$. The paper concludes in $\S 6$, where we describe some related open problems.

In addition to its intrinsics interest, this slightly simplified model will be used, together with experimental results, to identify the system parameters that are involved in the processes. Contact processes are notoriously difficult to observe directly, and the proposed setting makes their experimental determination easier, since the access to the contact patch is simpler. The results of the parameter identification will be used to identify the contact parameters.

Finally, we note that the contact area or patch between the wheel and the beam, as is explained in $\S 2$, has the shape of the beam's cross section but its location is unknown. This leads to a new free patch problem for the heat equation, which has been investigated in Kuttler \& Shillor [22]. 


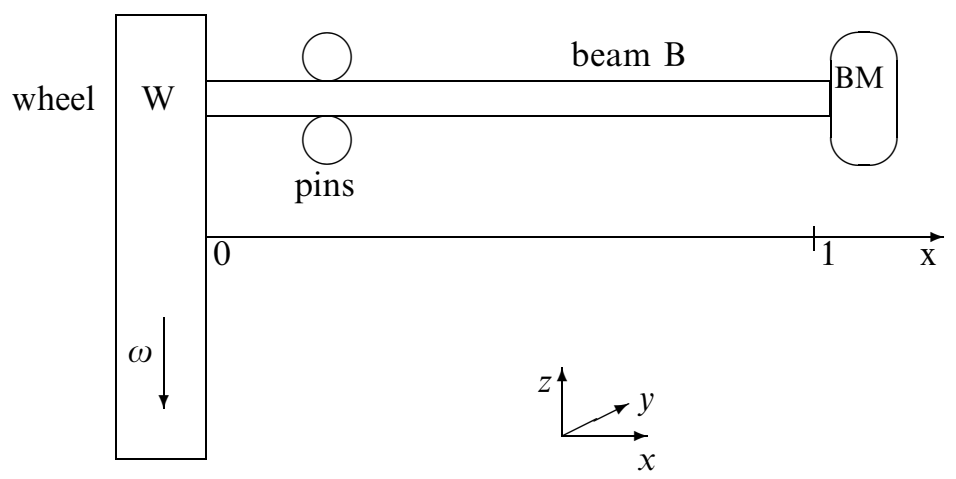

Figure 1. The physical setting.

\section{The model}

The physical setting consists of two beams, each one of which is rigidly attached at one end to a controlling mechanical device that causes the other end to come into frictional contact with a rotating wheel. As a result of the frictional traction the wheel slows down and eventually comes to a rest, releasing in the process its kinetic energy in the form of frictional heat. The wear of the contacting ends, which has an important effect on the process, is taken into account. This somewhat simplified setting allows us to investigate the braking process in an easier fashion, however, it is sufficiently realistic so we can compare its predictions with experimental results. The main simplifications are in replacing the braking pad with a beam, and in assuming that the wheel is rigid.

The device we have in mind includes two beams situated on both sides of the wheel, however, the setting is symmetric, so it is enough to consider one beam and one-half of the wheel, as depicted in Figure 1. The wheel $W$ is assumed to be rigid; it rotates about the $x$-axis with angular speed $\omega=\omega(t)$, has thickness $h_{w}$, radius $R$, mass $m$, moment of inertia $J$, and occupies the set

$$
W=\left\{(x, y, z):-h_{w} \leqslant x \leqslant 0, \sqrt{y^{2}+z^{2}} \leqslant R\right\} .
$$

The beam is parallel to the $x$-axis, in the $x z$-plane with $z=h$ and $0 \leqslant x \leqslant L$, i.e. in its stress free and isothermal reference configuration it occupies $B=\{(x, 0, z): 0 \leqslant x \leqslant$ $L, z=h\}$ and its cross section is $A_{b}$. The force $p_{*}=p_{*}(t)$ is applied by the controlling device (BM) at $x=L$, while at $x=0$ the beam is in contact with the wheel. Two rigid pins are situated above and below the beam restricting its motion to the line $L_{i}$ in the plane parallel to the $x y$-plane with $z=h$. Without this restriction, the motion of the contacting end would be in the $x=0$ plane too, complicating the model by introducing the need for two coupled beams, one vibrating in the $x y$-plane and the other in the $x z$-plane. Such a model will be considered in the sequel if warranted by experimental results.

We are interested in the dynamic braking process. Let $u=u(x, y, z, t)$ and $\theta=\theta(x, y, z, t)$ be the displacement (parallel to the $x y$-plane) and temperature fields of the beam, respectively. For the sake of convenience we use below the notation $u=u(x, t)$ and $\theta=\theta(x, t)$, since $y=0$ and $z=h$ are fixed. Although we consider the wheel as rigid (relative to the beam), we take into account its thermal characteristics, and denote by $\theta_{w}$ its temperature 


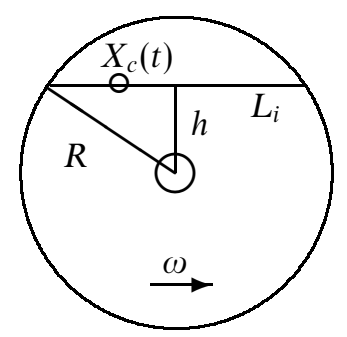

Figure 2. The beam's end $X_{c}(t)$ moves on the segment $L_{i}$.

field, which is defined on $W \times[0, T]$, for $T>0$. The angular velocity $\omega$ of the wheel depends on the frictional contact between the beam and the wheel, which is at the point

$$
X_{c}=X_{c}(t)=(x, y, z)=(0, u(0, t), h),
$$

and is restricted to move on the line $L_{i}$ such that $-l<y=u(0, t)<l$, where $l=\sqrt{R^{2}+h^{2}}$ (see Figure 2). The thermal expansion of the beam and the wear of its contacting end are included in the model, but since the wheel is assumed rigid we do not consider its wear.

We assume that the wear particles produced at the contacting end are instantly removed from the surface. We denote by $\xi=\xi(t)$ the shortening of the beam as a result of the wear; it measures the averaged length (over the beam's cross section $A_{b}$ ) of the removed material, and we refer the reader to $\mathrm{Gu}$ et al. [11] and $\mathrm{Gu} \&$ Shillor [12] for further details.

The beam is assumed viscoelastic and the shear stress is given by the Kelvin-Voigt constitutive relation

$$
-\sigma=-\sigma(x, t)=c^{2} u_{x x x}(x, t)+d c^{2} u_{t x x x}(x, t)
$$

where $\sigma(x, t)=\sigma(x, 0, h, t), c^{2}=E I /\left(\left|A_{b}\right| \rho\right)$ is the scaled elastic modulus, $E$ is the Young modulus, $\left|A_{b}\right|$ is the area of the cross section $A_{b}, I$ is the second moment of the cross section area, and $\rho$ is the material density. Also, $d(\geqslant 0)$ is the viscosity coefficient, and when $d=0$ the material is purely elastic. Here and below subscripts denote partial derivatives. The beam's equation of motion is

$$
u_{t t}+c^{2} u_{x x x x}+d c^{2} u_{t x x x x}=f
$$

where $f$ denotes the density (per unit length) of applied horizontal forces. In the process under consideration $f=0$, however, we retain it for the sake of generality.

It is well known that beams that vibrate with high frequency may generate considerable heat. However, in our setting the beam may vibrate only with low frequency, and so we neglected the term $\alpha_{B} \theta_{x}$ in (2.1) and $\alpha_{B} \theta_{x x}$ in (2.2).

The beam's energy equation is

$$
\theta_{t}-k_{B} \theta_{x x}=0
$$


where $k_{B}=\kappa / c\left|A_{b}\right| \rho$ is the (scaled) coefficient of thermal diffusivity; $\kappa$ is the coefficient of thermal conduction and $c$ is the heat capacity, both assumed positive constants.

We turn to describe the wheel. Let $\{\mathbf{i}, \mathbf{j}, \mathbf{k}\}$ denote the unit vectors in the directions of the coordinate axes, and let $\mathbf{r}=y \mathbf{j}+z \mathbf{k}$ denote the position of a point on the $x=0$ face of the wheel, for $|\mathbf{r}| \leqslant R$. Then, the energy equation of the wheel is

$$
\theta_{w, t}+\mathbf{v} \cdot \nabla \theta_{w}-k_{w} \Delta \theta_{w}=0
$$

where $k_{w}=\kappa_{w} / c_{w} \rho_{w}$ is the (scaled) coefficient of thermal diffusivity; $\kappa_{w}$ is the wheel's coefficient of thermal conduction and $c_{w}$ is its heat capacity. The velocity $\mathbf{v}$ is given by

$$
\mathbf{v}=\mathbf{r} \times \omega(t) \mathbf{i}
$$

and the term $\mathbf{v} \cdot \nabla \theta_{w}$ represent convective energy transport.

The beam's right end is rigidly attached to a controlling device which exerts a force $p_{*}$ in the (negative) $x$-direction, but does not move otherwise, thus $u(L, t)=u_{x}(L, t)=0$. The device's temperature is assumed to be $\theta_{d e v}=\theta_{d e v}(t)$, and we assume, for the sake of simplicity that the end has the device temperature, thus $\theta(L, t)=\theta_{d e v}(t)$. Choosing the heat exchange condition $\kappa \theta_{x}(L, t)=\gamma_{\text {dev }}\left(\theta_{\text {dev }}(t)-\theta(L, t)\right.$, leads to the same results as those below.

The wheel is held in place and a simple argument $[11,12]$ shows that the contact pressure caused by the horizontal thermal expansion, the wear and the applied force is given by

$$
p(t)=\left(p_{*}(t)+\alpha \int_{0}^{1} \theta(x, t) d x-\delta_{w} \xi(t)\right)_{+},
$$

where $f_{+}=\max \{f, 0\}, \alpha$ is the scaled coefficient of thermal expansion, and $\delta_{w}$ is the wear-pressure coefficient. The use of $(\cdot)_{+}$guarantees that the contact pressure vanishes when the beam is disengaged from the wheel. However, we note that in applications $p_{*}$ is kept at a sufficient level to prevent disengagement. The term $\delta_{w} \xi$ measures the pressure change because of length change resulting from wear, which although small, may or may not be negligible since it grows continuously and affects the process. When the applied force is large, we may neglect it, and the pressure resulting from thermal expansion as well. This simplifies the problem, and we remark on it in the sequel.

We now describe the frictional contact between the beam and the wheel. We note that when $\omega>0$ the wheel is rotating counterclockwise as seen from the beam. Moreover, the contact point between the beam and the wheel is at $\mathbf{r}=u(0, t) \mathbf{j}+h \mathbf{k}$, and the wheel velocity at this point is $\mathbf{v}=\omega h \mathbf{j}-\omega u \mathbf{k}$. Since the beam can move only horizontally, because of the restraining pins, the horizontal velocity of the contact point is $\omega h \mathbf{j}$, and therefore, the relative velocity between the beam's end and the contact point is

$$
\mathbf{v}_{\text {slip }}=\left(\omega h-u_{t}(0, t)\right) \mathbf{j}-\omega u(0, t) \mathbf{k}
$$

When the end's displacement is small (compared to $h$ ), we may approximate it by

$$
\mathbf{v}_{\text {slip }} \approx\left(\omega h-u_{t}(0, t)\right) \mathbf{j} .
$$


We model friction by a version of a general law, proposed in Strömberg et al. [32], which takes wear into account,

$$
\begin{gathered}
|\sigma(0, t)| \leqslant \mu H(t), \\
\frac{\sigma(0, t)}{\mu H(t)}=-\frac{\omega h-u_{t}(0, t)}{\left|\omega h-u_{t}(0, t)\right|} \quad \text { if } \quad u_{t}(0, t) \neq h \omega(t) .
\end{gathered}
$$

Here, $\mu$ denotes the coefficient of friction, which we discuss shortly; $\mu H$ is the friction bound, and $H$ is a function of the contact pressure, i.e. $H(t)=H(p(t))$. The following expression for $H$ has been derived in Strömberg et al. [32],

$$
H(p)=p(t)\left(1-\delta_{*} p(t)\right)_{+},
$$

where $\delta_{*}$ is a very small positive constant related to the surface wear and hardness. When $\delta_{*}=0$ we obtain $H(p)=p$, which is the usual choice for the Coulomb law. For the sake of generality, we assume here that $H$ is a nonnegative, bounded and Lipschitz continuous function of $p$. Moreover, the friction between the beam and the restraining pins is assumed negligible, otherwise it has to be included in the beam equation. On the other hand, because of the pins the friction condition involves only the component of the velocity in the $y$ direction $\omega h-u_{t}(0, t)$, since the pins supply the necessary force in the $z$ direction to cancel out any downward stress.

According to (2.7), when $\omega h \neq u_{t}(0, t)$ the end is in slip motion, in which $|\sigma|=\mu H(t)$ and the shear stress direction is opposite to the $y$-component of $\mathbf{v}_{\text {slip }}$. When $|\sigma|<\mu H$ the end sticks to the wheel and moves with it, which is stick motion. However, in our setting of the problem when the wheel is rotating, $\omega \neq 0$ and then $\mathbf{v}_{\text {slip }} \neq 0$ (except when $u(0, t)=0)$, and we have slip motion until the wheel comes to a complete stop.

We assume that the friction coefficient $\mu$ depends on the relative slip rate and on the surface temperature, which is often found in applications (e.g. see Rabinowicz [28] and the extensive references therein), thus

$$
\mu=\mu\left(\left|\mathbf{v}_{\text {slip }}(t)\right|, \theta(0, t)\right)
$$

where $\mu(\cdot, \cdot)$ is a prescribed, continuous and bounded function. Actually, this relationship holds true only if we assume that the tip temperature is the same as the wheel temperature at the contact point, which we do in this work. If the surface temperature of the wheel at the point is different from the beam's, then the dependence of $\mu$ on the temperature becomes very complicated. Indeed, in such a case one has to introduce the contact layer temperature, which we do not wish to pursue here.

For recent mathematical results and further references about slip rate and temperature dependence of $\mu$ the reader is referred elsewhere $[1,15,19,20,21,33,37]$, and the references therein.

The resultant of the moments acting on the contacting end is assumed to be zero. We next describe the thermal interaction between the beam's end and the wheel. The frictional power generated is

$$
P_{f}(t)=\mu H(t)\left|\mathbf{v}_{\text {slip }}\right|,
$$

i.e. the product of the friction traction and the relative slip rate. 
As is customary, we assume that a fraction $\gamma$ of the heat flux enters the beam and the rest flows into the wheel. The loss to the environment is assumed negligible, since the beam is thermally insulated lengthwise. Otherwise, all we need to do is to add a heat exchange term to the beam's energy equation. The thermal boundary condition at the contacting end is

$$
\kappa \theta_{x}(0, t)=-\gamma P_{f}(t)=-\gamma \mu H(t)\left|\mathbf{v}_{\text {slip }}\right| .
$$

The thermal boundary conditions on the wheel can be described in two ways. The more realistic one, which we employ in this work, assumes that the contact, seen from the wheel, takes place over a patch of cross section $A_{b}$ centered at $X_{c}$, which we denote by $\mathscr{A}_{t}$, i.e. the thickness of the beam is taken into account. The idealized option is to assume that the contact is just at the point $X_{c}$, however, we do not consider this case here.

Thus, we assume that the boundary $\partial W$ of the wheel has two components $\Gamma_{e}$ and $\Gamma_{c}$, where

$$
\Gamma_{c}=\Gamma_{c}(t)=\left\{(0, y, z):(y, z) \in \mathscr{A}_{t}\right\},
$$

and $\Gamma_{e}=\partial W-\bar{\Gamma}_{c}(t)$. Both $\Gamma_{c}$ and $\Gamma_{e}$ are process dependent, and in a sense, their common boundary $\Gamma_{F}=\partial \bar{\Gamma}_{e} \cap \partial \bar{\Gamma}_{c}$ is a free boundary, whose shape is known but location unknown, beforehand.

In particular, if the beam's cross-section $A_{b}$ is circular with radius $r_{c}$, then

$$
\Gamma_{c}=\Gamma_{c}(t)=\left\{(0, y, z): \sqrt{(y-u(0, t))^{2}+(z-h)^{2}}<r_{c}\right\} .
$$

The wheel exchanges energy with the environment over $\Gamma_{e}$, thus

$$
\kappa_{w} \frac{\partial \theta_{w}}{\partial n}=h_{w}^{*}\left(\theta_{a m b}-\theta_{w}\right)
$$

where $\theta_{a m b}$ is the ambient temperature, $h_{w}^{*}$ is the heat exchange coefficient, $\mathbf{n}$ is the outer normal to $W$ and $\partial / \partial n$ is the normal derivative.

On $\Gamma_{c}$, the normal is $\mathbf{n}=\mathbf{i}$ and the boundary condition is

$$
\kappa_{w} \frac{\partial \theta_{w}}{\partial x}=\frac{(1-\gamma)}{\left|A_{b}\right|} P_{f}(t)=\frac{(1-\gamma)}{\left|A_{b}\right|} \mu H(t)\left|\mathbf{v}_{\text {slip }}\right|,
$$

where, for the sake of simplicity, we scaled the problem so that $\left|A_{b}\right|=1$.

We note that the $z$ component of the torque at the beam's end vanishes because of the pins, and therefore, the torque balance is

$$
J \frac{d \omega}{d t}=h \sigma(0, t)
$$

where $\sigma(0, t)$ satisfies (2.7). Let $\psi(r)=|r|$, and denote by $\partial \psi$ its subdifferential, i.e.

$$
\partial \psi(r)=\left\{\begin{array}{cc}
1 & 0<r \\
{[-1,1]} & r=0 \\
-1 & r<0
\end{array}\right.
$$

then the boundary condition corresponding to the mechanical contact of the end of the 
beam and the wheel becomes the differential equation,

$$
J \frac{d \omega}{d t}=-h \mu H(p) \partial \psi\left(h \omega-u_{t}\right) .
$$

When there is stick the moment acting on the wheel belongs to the interval $[-h \mu H, h \mu H]$, while in slip state the acting moment is $\pm h \mu H$. Note that the vibrations of the end of the beam may either increase or decrease the angular velocity.

Finally, the wear of the beam's end is given by the differential form of the Archard law:

$$
\frac{d \xi}{d t}=k_{w e} p(t)\left|\mathbf{v}_{s l i p}\right|
$$

where $k_{w e}$ is the wear coefficient (very small in practice), and the wear rate is proportional to the contact pressure and slip rate (e.g. see elsewhere [4, 5, 11, 32, 37], and the references therein).

Initially, $u(x, 0)=u_{0}(x), u_{t}(x, 0)=v_{0}(x)$ and $\theta(x, 0)=\theta_{0}(x)$, for $0 \leqslant x \leqslant 1$, where $u_{0}, v_{0}$ and $\theta_{0}$ represent the beam displacement, velocity and temperature, respectively. The initial wheel temperature is $\theta_{w 0}$ on $W$ and its angular velocity is $\omega(0)=\omega_{0}$.

We set $L=1$ and use the notation $\Omega_{T}=(0,1) \times(0, T), W_{T}=W \times(0, T), \Gamma_{e T}=\Gamma_{e} \times$ $(0, T)$ and $\Gamma_{c T}=\Gamma_{c} \times(0, T)$.

Collecting the equations and conditions above leads to the following classical statement of the thermomechanical problem of the braking of a rotating wheel by frictional contact with a beam.

Find a set of functions $\left\{u, \theta, \omega, \xi, \theta_{w}\right\}$ such that:

$$
\begin{array}{rlrl}
u_{t t}+c^{2} u_{x x x x}+c^{2} d u_{t x x x x} & =f, & & \text { in } \Omega_{T}, \\
\theta_{t}-k_{B} \theta_{x x} & =0, & & \text { in } \Omega_{T}, \\
u(x, 0)=u_{0}(x), \quad u_{t}(x, 0) & =v_{0}(x), & & 0 \leqslant x \leqslant 1, \\
\theta(x, 0) & =\theta_{0}(x), & 0 \leqslant x \leqslant 1, \\
u(1, t)=0, & u_{x}(1, t) & =0, & \theta(1, t)=\theta_{d e v}(t), \\
\theta_{w, t}+\mathbf{v} \cdot \nabla \theta_{w}-k_{w} \Delta \theta_{w} & =0, & & \text { in } W_{T}, \\
\mathbf{v} & =\mathbf{r} \times \omega(t) \mathbf{i}, & & \text { in } W_{T}, \\
\kappa_{w} \frac{\partial \theta_{w}}{\partial n} & =h_{w}^{*}\left(\theta_{a m b}-\theta_{w}\right), \quad \text { on } \Gamma_{e T}, \\
\kappa_{w} \theta_{w, x} & =(1-\gamma) \mu H(p)\left|\mathbf{v}_{s l i p}\right|, \quad \text { on } \Gamma_{c T} \\
\theta_{w}(\cdot, 0) & =\theta_{0 w} & & \text { on } W,
\end{array}
$$

and at $x=0$,

$$
\begin{aligned}
u_{x x}+d u_{t x x} & =0, \\
\kappa \theta_{x} & =-\gamma \mu H(p)\left|\mathbf{v}_{\text {slip }}\right|, \\
|\sigma| & \leqslant \mu H(p), \\
\sigma & =-\mu H(p), \quad \text { if }\left|\omega h-u_{t}\right| \neq 0, \\
J \frac{d \omega}{d t} & =-h \mu H(p) \partial \psi\left(h \omega-u_{t}\right),
\end{aligned}
$$




$$
\begin{aligned}
\frac{d \xi}{d t} & =k_{w e} p(t)\left|\mathbf{v}_{\text {slip }}\right|, \\
\omega(0) & =\omega_{0}, \quad \xi(0)=\xi_{0},
\end{aligned}
$$

for $0 \leqslant t \leqslant T$. Here, the wheel and beam temperatures are equal at the contact point, $\mu=\mu\left(\left|\mathbf{v}_{\text {slip }}\right|, \theta\right)$ and $H(p)=H(p(t))$, where $p$ is given by (2.6).

Note that the coupling between the mechanical and thermal processes is through $\mathbf{v}$ in (2.13) and (2.14), the boundary conditions (2.16), (2.19) and via $\mu$ and $p$, introducing strong nonlinearities into the problem. Moreover, $\Gamma_{c}$ is related to one of the unknowns of the problem $(u(0, t))$.

A number of simplifications are possible, making the problem easier to handle both mathematically and numerically. The contribution of the term $\delta_{w} \xi(t)$ to the pressure $p$ is likely to be small, so we may neglect it. Moreover, by controlling the applied pressure we may replace $p$ with $p_{*}$ in the model. This decouples the wear process and allows to compute the accumulated wear separately, once the solution of the rest of the problem has been obtained. However, for the sake of generality, we consider the fully coupled problem.

Since the problem includes friction, in general it may have no classical solutions [7]. Therefore, it is necessary to consider the weak formulation of the problem. Moreover, weak formulations provide a natural setting for the FEM algorithm for the problem.

\section{Weak formulation, statement of results}

We turn to the weak or variational formulation of the problem. Then we describe the assumptions on the problem data, and recall the existence (Theorem 3.2) and the uniqueness (Theorem 3.3) results established in Kuttler et al. [23]. We refer the reader elsewhere [7, 17, 24] for details on the function spaces which we will use in this and the following sections.

First, we redefine the dependent variables and assume, without loss of generality, that $\theta_{\text {dev }}=\theta_{\text {amb }}=0$. We let $u$ and $\theta$ lie in the spaces

$$
V \equiv\left\{u \in H^{2}(0,1): u_{x}(1)=u(1)=0\right\}, \quad E \equiv\left\{\eta \in H^{1}(0,1): \eta(1)=0\right\},
$$

respectively. We note that the norm $\|u\|_{V}=\left|u_{x x}\right|_{L^{2}(0,1)}$ is equivalent to the usual norm on $V$, and $\|\theta\|_{E}=\left|\theta_{x}\right|_{L^{2}(0,1)}$ is equivalent to the usual norm on $E$. We use these equivalent norms whenever convenient. We denote by $\gamma_{0}$ the trace map of an element of $V$ at $x=0$, i.e. $\gamma_{0} v=v(0)$, and denote by $\gamma_{w}$ the trace map from $H^{1}(W)$ to $L^{2}(\partial W)$.

We define $H \equiv L^{2}(0,1)$ and identify $H$ and $H^{\prime}$ so that

$$
E \subseteq H=H^{\prime} \subseteq E^{\prime}
$$

Similar set inclusions hold when $E$ is replaced by $V$. We also assume that $\omega$ and $\xi$ lie in $L^{\infty}(0, T)$. For convenience, we denote by $\mathscr{V}=L^{2}(0, T ; V)$ and $\mathscr{E}=L^{2}(0, T ; E)$. We seek $\theta_{w}$ in the space $\mathscr{V}_{w} \equiv L^{2}\left(0, T ; H^{1}(W)\right)$. 
Derivation of the weak formulation

The derivation may be skipped on first reading, and the reader may want to proceed directly to the formulation.

The weak formulation of the problem is obtained by multiplying the equations with appropriate test functions and using integration by parts and the given boundary conditions. In such a way we obtain operators mapping Banach spaces into their duals.

We begin with (2.8), which we rewrite in terms of $v=u_{t}$, and so

$$
u(x, t)=u_{0}(x)+\int_{0}^{t} v(x, s) d s .
$$

Multiplying both sides of (2.8) by $w \phi$, where $w \in V$ and $\phi \in C_{c}^{\infty}(0, T)$ and integrating by parts, using $\sigma=-c^{2}\left(u_{x x x}+d u_{t x x x}\right)$, and another integration by parts yields

$$
\begin{aligned}
& -\int_{0}^{T} \int_{0}^{1} v w \phi_{t} d x d t+\int_{0}^{T} \sigma(0, t) w(0) \phi(t) d t+\int_{0}^{T} \int_{0}^{1}\left(c^{2} u_{x x}+d c^{2} v_{x x}\right) w_{x x} \phi d x d t \\
& =\int_{0}^{T} \int_{0}^{1} f w \phi d x d t .
\end{aligned}
$$

Formally, conditions (2.20) and (2.21) imply that

$$
\sigma(0, t) \in \mu\left(\left|\mathbf{v}_{\text {slip }}\right|, \theta\right) H(p) \partial \psi(v-h \omega) .
$$

Thus, we obtain the following variational formulation of the equation and the boundary conditions for $u$ :

$$
\begin{aligned}
& -\int_{0}^{T} \int_{0}^{1} v w \phi_{t} d x d t+\int_{0}^{T} \mu H(p) \zeta(t) w(0) \phi(t) d t \\
& \quad+\int_{0}^{T} \int_{0}^{1}\left(c^{2} u_{x x}+d c^{2} v_{x x}\right) w_{x x} \phi d x d t=\int_{0}^{T} \int_{0}^{1} f w \phi d x d t
\end{aligned}
$$

Here, $\zeta(t) \in L^{\infty}(0, T), \sigma(0, t)=\mu H \zeta$, and $\zeta \in \partial \psi(v-h \omega)$, so, for all $\eta \in L^{\infty}(0, T)$,

$$
\int_{0}^{T} \zeta(t) \eta(t) d t \leqslant \int_{0}^{T}\left(\left|\gamma_{0} v(t)-h \omega(t)+\eta(t)\right|-\left|\gamma_{0} v(t)-h \omega(t)\right|\right) d t .
$$

We define the set-valued map,

$$
Q: L^{2}(0, T) \times L^{2}(0, T) \times \mathscr{V} \times L^{2}(0, T) \rightarrow \mathscr{P}\left(\mathscr{V}^{\prime}\right)=\mathscr{P}\left(L^{2}\left(0, T ; V^{\prime}\right)\right)
$$

as follows: $y^{*} \in Q(\mu, p, v, \omega)$ if and only if there exists $\zeta \in L^{\infty}(0, T)$ such that (3.7) holds for all $\eta \in L^{2}(0, T)$ and $y^{*}(t)=\gamma_{0}^{*}(\mu H(p) \zeta)(t)$ a.e. in $(0, T)$.

We define the operator $A: V \rightarrow V^{\prime}$ by $\langle A u, w\rangle \equiv \int_{0}^{1} u_{x x} w_{x x} d x$, and will also regard it as an operator from $\mathscr{V}$ to $\mathscr{V}^{\prime}$ by setting $A u(t) \equiv A(u(t))$.

Next, we consider (2.13), and note that on $\Gamma_{e T}$ a homogeneous Neumann condition holds, while on $\Gamma_{c T}$ the frictional heat flux condition holds. Let $\phi \in C_{c}^{\infty}(0, T)$ and 
$\beta \in H^{1}(W)$. We multiply (2.13) by $\beta \phi$ and integrate by parts, which yields

$$
\begin{aligned}
& -\int_{0}^{T} \int_{W} \theta_{w} \beta \phi^{\prime} d V d t+\int_{0}^{T} \int_{W} \mathbf{v} \cdot \nabla \theta_{w} \beta \phi d V d t \\
& \quad+\int_{0}^{T} \int_{W} k_{w} \nabla \theta_{w} \cdot \nabla(\beta) \phi d V d t-\int_{0}^{T} \int_{\partial W} k_{w} \frac{\partial \theta_{w}}{\partial n} \beta \phi d S d t=0 .
\end{aligned}
$$

We define the operator $A_{w}: H^{1}(W) \rightarrow H^{1}(W)$ by $\left\langle A_{w} \theta_{w}, \sigma_{w}\right\rangle \equiv \int_{W} \nabla \theta_{w} \cdot \nabla \sigma_{w} d V$, and let $A_{w}: \mathscr{V}_{w} \rightarrow \mathscr{V}_{w}^{\prime}$ be its extension, given by $A_{w} \theta_{w}(t) \equiv A_{w}\left(\theta_{w}(t)\right)$.

Recalling that $\theta_{a m b}=0$, we get from (3.8)

$$
\begin{aligned}
& -\int_{0}^{T} \int_{W} \theta_{w} \beta \phi^{\prime} d V d t+\int_{0}^{T} \int_{W} \mathbf{v} \cdot \nabla \theta_{w} \beta \phi d V d t+\int_{0}^{T} \int_{W} k_{w} \nabla \theta_{w} \cdot \nabla \beta \phi d V d t \\
& +\int_{0}^{T} \int_{\Gamma_{e}(t)} h_{w} \theta_{w} \beta \phi d S d t-(1-\gamma) \int_{0}^{T} \int_{\Gamma_{c}(t)} \mu H(p)\left|\mathbf{v}_{s l i p}\right| \beta \phi d S d t=0
\end{aligned}
$$

We note that $\Gamma_{c}(t)$ and $\Gamma_{e}(t)$ depend on $u(0, t)$, and the last two terms on the left-hand side are highly nonlinear.

We now introduce the additional operators $N_{1}, N_{2}$ and $N$. Let $\theta_{w} \in L^{2}\left(0, T ; H^{1}(W)\right)$ and $u \in C(0, T ; V)$. The nonlinear operator

$$
N_{1}: L^{2}\left(0, T ; H^{1}(W)\right) \times C(0, T ; V) \rightarrow \mathscr{V}_{w}^{\prime},
$$

is defined by

$$
\left\langle N_{1}\left(\theta_{w}, u\right), \sigma_{w}\right\rangle \equiv \int_{0}^{T} \int_{\Gamma_{e}\left(\gamma_{0} u(t)\right)} h_{w} \gamma_{w} \theta_{w} \gamma_{w} \sigma_{w} d S d t
$$

and

$$
N_{2}: L^{2}(0, T) \times L^{2}(0, T) \times L^{2}(0, T ; V) \times C(0, T) \times C(0, T ; V) \rightarrow \mathscr{V}_{w}^{\prime},
$$

is given by

$$
\left\langle N_{2}(\mu, p, v, \omega, u), \sigma_{w}\right\rangle \equiv \int_{0}^{T} \int_{\Gamma_{c}\left(\gamma_{0} u(t)\right)}(1-\gamma) \mu H(p)\left|\mathbf{v}_{s l i p}\right| \gamma_{w} \sigma_{w} d S d t
$$

where $\mathbf{v}_{\text {slip }}=\left(\gamma_{0} v-h \omega\right) \mathbf{j}$. Then, $N$ is defined as

$$
N\left(\theta_{w}, \mu, p, v, \omega, u\right) \equiv N_{1}\left(\theta_{w}, u\right)-N_{2}(\mu, p, v, \omega, u)
$$

Recalling that $\mathbf{v}=\mathbf{r} \times \omega \mathbf{i}$, the second term in (3.9) may be described in terms of the operator $N_{3}: \mathscr{V}_{w} \times C(0, T) \rightarrow \mathscr{V}^{\prime}{ }_{w}$, defined as

$$
\left\langle N_{3}\left(\theta_{w}, \omega\right), \sigma_{w}\right\rangle \equiv \int_{0}^{T} \int_{W}(\mathbf{r} \times \omega \mathbf{i}) \cdot \nabla \theta_{w} \sigma_{w} d V d t
$$

where $\mathbf{r}=(0, y, z)$ for a point $(x, y, z) \in W$, and $\omega$ satisfies (2.22). 
Motivated by the differential inclusion (2.22), we define $Q_{1}:\left(L^{2}(0, T)\right)^{4} \rightarrow \mathscr{P}\left(L^{2}(0, T)\right)$ as follows:

$$
\lambda \in Q_{1}(\mu, p, \omega, v),
$$

if and only if there exists $\zeta_{1} \in L^{2}(0,1)$ such that for all $\eta \in L^{2}(0, T)$ we have

$$
\int_{0}^{T} \zeta_{1}(t) \eta(t) d t \leqslant \int_{0}^{T}(|h \omega(t)-v(0, t)+\eta(t)|-|h \omega(t)-v(0, t)|) d t,
$$

and $\lambda=h \mu H(p) \zeta_{1}$.

We now write (2.9), along with the associated boundary and initial conditions, as

$$
-\int_{0}^{T} \int_{0}^{1} \theta \phi^{\prime} \eta d x d t+k_{B} \int_{0}^{T} \int_{0}^{1} \theta_{x} \eta_{x} \phi d x d t-\int_{0}^{T} \gamma \mu H(p)\left|\mathbf{v}_{s l i p}\right| \gamma_{0} \eta \phi d t=0,
$$

where $\phi \in C_{c}^{\infty}(0, T)$ and $\eta \in E$. We define $L: E \rightarrow E^{\prime}$ by $\langle L \theta, \eta\rangle \equiv \int_{0}^{1} \theta_{x} \eta_{x} d x$, and $N_{4}$ : $L^{2}(0, T)^{4} \times C(0, T) \rightarrow \mathscr{E}^{\prime}$ by

$$
\left\langle N_{4}(\mu, p, v, \omega, u), \eta\right\rangle \equiv \int_{0}^{T} \gamma \mu H(p)\left|h \omega-\gamma_{0} v\right| \gamma_{0} \eta d t .
$$

\section{End of derivation}

We now write the weak formulation of the thermomechanical problem (2.8)-(2.24) in terms of these operators. The dynamic initial-boundary value problem for the beam may be written as

$$
v^{\prime}+c^{2} A u+d c^{2} A v+Q(\mu, p, v, \omega) \ni f \quad \text { in } \mathscr{V}^{\prime}, \quad v(0)=v_{0},
$$

with $u(t)=u_{0}+\int_{0}^{t} v(s) d s$. The initial-boundary value problem for the temperature of the rotating wheel is

$$
\begin{gathered}
\theta_{w}^{\prime}+N_{3}\left(\theta_{w}, \omega\right)+k_{w} A_{w} \theta_{w}+N_{1}\left(\theta_{w}, \gamma_{0} u\right)-N_{2}\left(\mu, p, v, \omega, \gamma_{0} u\right)=0, \\
\theta_{w}(0)=\theta_{w 0} .
\end{gathered}
$$

The initial-boundary value problem for the beam temperature is

$$
\theta^{\prime}+k_{B} L \theta+N_{4}(\mu, p, v, \omega, u)=0, \quad \theta(0)=\theta_{0} .
$$

The initial value problem for the angular velocity $\omega$ is

$$
\omega^{\prime}+J^{-1} Q_{1}(\mu, p, v, \omega) \ni 0, \quad \omega(0)=\omega_{0} .
$$

Finally, the wear is obtained by solving the initial value problem

$$
\xi^{\prime}=k_{w e} p\left|\mathbf{v}_{\text {slip }}\right|, \quad \xi(0)=\xi_{0} .
$$

We recall that $\mu(t)=\mu\left(\left|\mathbf{v}_{\text {slip }}(t)\right|, \gamma_{0} \theta(t)\right)$, and

$$
p(t)=\left(p_{*}(t)+\alpha \int_{0}^{1} \theta(x, t) d x-\delta_{w} \xi(t)\right)_{+} .
$$


Next, we rewrite the weak formulation in a condensed way. Let

$$
Y \equiv\left(V \times H^{1}(W) \times E \times \mathbb{R} \times \mathbb{R}\right)
$$

Problem 3.1 Find the vector function $\mathbf{y}=\left(v, \theta_{w}, \theta, \omega, \xi\right) \in Y$ such that

$$
\mathbf{y}^{\prime}+\mathscr{2} \mathbf{y}+\mathscr{N} \mathbf{y}+\mathscr{A} \mathbf{y} \ni \mathbf{f}, \quad \mathbf{y}(0)=\mathbf{y}_{0}
$$

where

$$
\mathbf{y}_{0}=\left(v_{0}, \theta_{w_{0}}, \theta_{0}, \omega_{0}, \xi_{0}\right) \in L^{2}(0,1) \times L^{2}(W) \times L^{2}(0,1) \times \mathbb{R}_{+} \times \mathbb{R}_{+},
$$

is the vector of initial conditions.

The operators $\mathscr{Q}, \mathscr{N}$ and $\mathscr{A}$ map $L^{2}(0, T ; Y)$ to $L^{2}\left(0, T ; Y^{\prime}\right)$ or $\mathscr{P}\left(L^{2}\left(0, T ; Y^{\prime}\right)\right)$, and are defined as follows. Let $\mathbf{z} \equiv\left(w, \sigma_{w}, \phi, \eta, \omega\right)$, then

$$
\begin{aligned}
\langle\mathscr{2} \mathbf{y}, \mathbf{z}\rangle & \equiv\langle Q(\mu, p, v, \omega), v\rangle+\frac{1}{J}\left\langle Q_{1}(\mu, p, v, \omega), \eta\right\rangle \\
& =\int_{0}^{T} \mu H(p) \zeta \gamma_{0} w d t+\frac{1}{J} \int_{0}^{T} h \mu H(p) \zeta_{1} \eta d t .
\end{aligned}
$$

where $\zeta$ and $\zeta_{1}$ satisfy (3.7) and (3.15), respectively.

$$
\begin{aligned}
\langle\mathscr{N} \mathbf{y}, \mathbf{z}\rangle \equiv & \left\langle N_{1}\left(\theta_{w}, \gamma_{0} u\right), \sigma_{w}\right\rangle-\left\langle N_{2}\left(u, p, v, \omega, \gamma_{0} u\right), \sigma_{w}\right\rangle \\
& +\left\langle N_{3}\left(\theta_{w}, \omega\right), \sigma_{w}\right\rangle+\left\langle N_{4}(\mu, p, \omega, u), \phi\right\rangle .
\end{aligned}
$$

Finally,

$$
\langle\mathscr{A} \mathbf{y}, \mathbf{z}\rangle \equiv c^{2}\langle A u, w\rangle+c^{2} d\langle A v, w\rangle+k_{w}\left\langle A_{w} \theta_{w}, \sigma_{w}\right\rangle+k_{B}\langle L \theta, \phi\rangle .
$$

We note that the time derivative of $\mathbf{y}$ in (3.25) is defined in the sense of $Y^{\prime}$ valued distributions.

We make the following assumptions on the problem data.

\section{Assumptions}

(1) $H(\cdot)$ is a bounded, positive and Lipschitz function;

(2) $\mu(\cdot, \cdot)$ is a bounded, positive and Lipschitz function in both variables, and

$$
0<\mu_{*} \leqslant \mu \leqslant \mu^{*}
$$

(3) $u_{0} \in V, \quad v_{0} \in L^{2}(0,1) \quad \theta_{0} \in L^{2}(0,1), \quad \theta_{w 0} \in L^{2}(W)$;

(4) $\theta_{\text {dev }}=0, \quad \theta_{\text {amb }}=0$;

(5) $0<\omega_{0}, \quad 0 \leqslant \xi_{0}$.

The following existence result for Problem 3.1 has been established in Kuttler et al. [23].

Theorem 3.2 Assume that the data satisfy assumptions 1-5. Then there exists $\mathbf{y} \in Y$ such that (3.25) holds. 
The proof was based on the recent results, established in Kuttler \& Shillor [19] (see also Kuttler [18]), on evolution equations involving set-valued pseudomonotone operators.

We conclude that problem (2.8)-(2.24) has a weak solution.

The uniqueness of the solution is an open problem. A partial result has been provided [23], where it was shown that the weak solution is unique when the angular velocity $\omega$ and the temperature $\theta_{w}$ of the wheel are known. We present this result here since it will be used in the future in the parameter identification of the system coefficients. Indeed, once the wheel temperature and its angular speed are obtained from measurements, the uniqueness of the weak solution will guarantee that the optimization involved in parameter identification will produce reliable results.

Theorem 3.3 If assumptions 1-5 hold and $\theta_{w}$ and $\omega$ are given functions, then problem (3.18), (3.21) and (3.23), in which $N_{4}$ is given by (3.17), admits a unique solution.

\section{Numerical algorithm}

In this section a fully-discrete approximation scheme of the variational problem is described. Since our interest lies in the process when the rotating wheel comes to a stop, we assume that there is only slip between the beam tip and the wheel. This removes condition (2.20), friction is represented by (2.21), and (3.5) becomes an equation. We, in addition, neglect the last term in (2.6).

We assume that $L=1$ and let $0=x_{0}<x_{1}<\ldots<x_{M}=1$, be a partition of [0,1], with $I_{i}=\left[x_{i-1}, x_{i}\right]$ and $h_{i}=x_{i}-x_{i-1}$ for $i=1, \ldots, M$, and $h=\max _{1 \leqslant i \leqslant M} h_{i}$ denotes the maximal discretization step size. Following Han et al. [14], we define the finite element spaces approximating the variational spaces $V$ and $E$ :

$$
\begin{aligned}
& V^{h}=\left\{v^{h} \in V ; \quad v_{\left.\right|_{I_{i}}}^{h} \text { is cubic, } \quad 1 \leqslant i \leqslant M\right\}, \\
& E^{h}=\left\{\xi^{h} \in E ; \quad \xi_{\left.\right|_{I_{i}}}^{h} \text { is affine, } 1 \leqslant i \leqslant M\right\} .
\end{aligned}
$$

That is, $V^{h}$ and $E^{h}$ are composed of $C^{1}$ piecewise cubic and continuous piecewise affine functions, respectively.

$W$ is assumed to be a polyhedral domain and let $\mathscr{T}^{h}$ be a finite element partition of $\bar{W}$ compatible with the boundary splitting $\Gamma=\Gamma_{e} \cup \Gamma_{c}$. We use the following finite element space $B^{h}$ to approximate $H^{1}(W)$,

$$
B^{h}=\left\{\Phi^{h} \in C(\bar{W}) ; \quad \Phi_{\left.\right|_{\kappa}}^{h} \in P_{1}(\kappa), \quad \forall \kappa \in \mathscr{T}^{h}\right\},
$$

where $P_{1}(\kappa)$ denotes the space of polynomials of global degree at most one in $\kappa$. Here, $h$ represents the spatial discretization parameter of $W$.

To approximate the time derivatives, we introduce a uniform partition $0=t_{0}<t_{1}<\ldots<$ $t_{N}=T$ of the time interval $[0, T]$, and let $k$ be the time step size. For a continuous function $w(t)$ we let $w_{n}=w\left(t_{n}\right)$ and, for a sequence $\left\{w_{n}\right\}_{n=0}^{N}, \delta w_{n}=\left(w_{n}-w_{n-1}\right) / k$ denotes divided differences.

A fully discrete scheme for the problem is as follows. 
Find $v^{h k}=\left\{v_{n}^{h k}\right\}_{n=0}^{N} \subset V^{h}, \theta^{h k}=\left\{\theta_{n}^{h k}\right\}_{n=0}^{N} \subset E^{h}, \theta_{w}^{h k}=\left\{\theta_{w n}^{h k}\right\}_{n=0}^{N} \subset B^{h},\left\{\omega_{n}^{h k}\right\}_{n=0}^{N}$ and $\left\{\xi_{n}^{h k}\right\}_{n=0}^{N}$ such that:

$$
u_{0}^{h k}=u_{0}^{h}, \quad v_{0}^{h k}=v_{0}^{h}, \quad \theta_{0}^{h k}=\theta_{0}^{h}, \quad \theta_{w, 0}^{h k}=\theta_{w, 0}^{h}, \quad \omega_{0}^{h k}=\omega_{0}^{h}, \quad \xi_{0}^{h k}=\xi_{0}^{h},
$$

and for $n=1,2, \ldots, N$ :

$$
\begin{aligned}
& \int_{0}^{1} \delta v_{n}^{h k} w^{h} d x+c^{2} d \int_{0}^{1}\left(v_{n}^{h k}\right)_{x x} w_{x x}^{h} d x=\int_{0}^{1} f_{n} w^{h} d x \\
& -c^{2} \int_{0}^{1}\left(u_{n-1}^{h k}\right)_{x x} w_{x x}^{h} d x+\mu_{n-1} H\left(p_{n-1}^{h k}\right) w^{h}(0), \quad w^{h} \in V^{h}, \\
& J \delta \omega_{n}^{h k}=-h \mu_{n-1} H\left(p_{n-1}^{h k}\right), \\
& \delta \xi_{n}^{h k}=k_{w e} p_{n-1}^{h k}\left|v_{n}^{h k}(0)-h \omega_{n}^{h k}\right|, \\
& \int_{0}^{1} \delta \theta_{n}^{h k} \phi^{h} d x+k_{B} \int_{0}^{1}\left(\theta_{n}^{h k}\right)_{x} \phi_{x}^{h} d x=\gamma \mu_{n-1} H\left(p_{n-1}^{h k}\right)\left|v_{n}^{h k}(0)-h \omega_{n}^{h k}\right| \phi^{h}(0), \quad \phi^{h} \in E^{h}, \\
& \int_{W} \delta \theta_{w, n}^{h k} \Phi^{h} d \boldsymbol{x}+\int_{W} w_{n} \boldsymbol{v} \cdot \nabla \theta_{w, n-1}^{h k} \Phi^{h} d \boldsymbol{x}+\int_{W} k_{w} \nabla \theta_{w, n}^{h k} \nabla \Phi^{h} d \boldsymbol{x} \\
& \quad+\int_{\Gamma_{e}}^{h_{w}} \theta_{w, n}^{h k} \Phi^{h} d S-(1-\gamma) \int_{\Gamma_{c}} \mu_{n-1} H\left(p_{n-1}^{h k}\right)\left|v_{n}^{h k}(0)-h \omega_{n}^{h k}\right| \Phi^{h} d S=0, \quad \Phi^{h} \in B^{h}, \\
& p_{n}^{h k}=\left(\left(p_{*}\right)_{n}+\alpha \sum_{i=1}^{M} l_{i} \theta_{n}^{h k}\left(x_{i}\right)-\delta_{w} \xi_{n}^{h k}\right)_{+}, \\
& u_{n}^{h k}=u_{n-1}^{h k}+k v_{n}^{h k},
\end{aligned}
$$

where $\mu_{n-1}=\mu\left(\left|\left(v_{s l i p}\right)_{n-1}\right|, \theta_{n-1}^{h k}\right),\left(v_{s l i p}\right)_{n-1}=\omega_{n-1}^{h k} h-v_{n-1}^{h k}(0)$, and $p_{0}^{h k}$ is defined by

$$
p_{0}^{h k}=\left(\left(p_{*}\right)_{0}+\alpha \sum_{j=1}^{M} h_{i} \theta_{0}^{h k}\left(x_{i}\right)-\delta_{w} \xi_{0}^{h k}\right)_{+},
$$

$h_{i}=x_{i}-x_{i-1}$ is the size of the element $I_{i}, \boldsymbol{v}=(0, z,-y)$, and $u_{0}^{h}, v_{0}^{h}, \theta_{0}^{h}, \theta_{w, 0}^{h}, \omega_{0}^{h}$ and $\xi_{0}^{h}$ are appropriate approximations of $u_{0}, v_{0}, \theta_{0}, \theta_{w, 0}, \omega_{0}$ and $\xi_{0}$, respectively.

We choose the friction coefficient as

$$
\mu(v, \theta)=\frac{1}{2} \lambda_{1} e^{-\lambda_{2} v}\left(1+e^{-\lambda_{3} \theta}\right)
$$

where $\lambda_{1}, \lambda_{2}, \lambda_{3}>0$, which is a decreasing function of the slip rate $v$ and increasing with the temperature $\theta$.

We conclude this section by describing briefly how to solve the fully discrete problem. We suppose that $n \in\{1, \ldots, N\}$ and $u_{n-1}^{h k}, v_{n-1}^{h k}, \omega_{n-1}^{h k}, \xi_{n-1}^{h k}, \theta_{n-1}^{h k}$ and $\theta_{w, n-1}^{h k}$ have already been computed.

First, we note that variational equality (4.1) may be written as

$$
\begin{aligned}
& \int_{0}^{1} v_{n}^{h k} w^{h} d x+c^{2} d k \int_{0}^{1}\left(v_{n}^{h k}\right)_{x x} w_{x x}^{h} d x=k \int_{0}^{1} f_{n} w^{h} d x \\
& +c^{2} k \int_{0}^{1}\left(u_{n-1}^{h k}\right)_{x x} w_{x x}^{h} d x-v k H\left(p_{n-1}\right) w^{h}(0)+\int_{0}^{1} v_{n-1}^{h k} w^{h} d x, \quad w^{h} \in V^{h},
\end{aligned}
$$



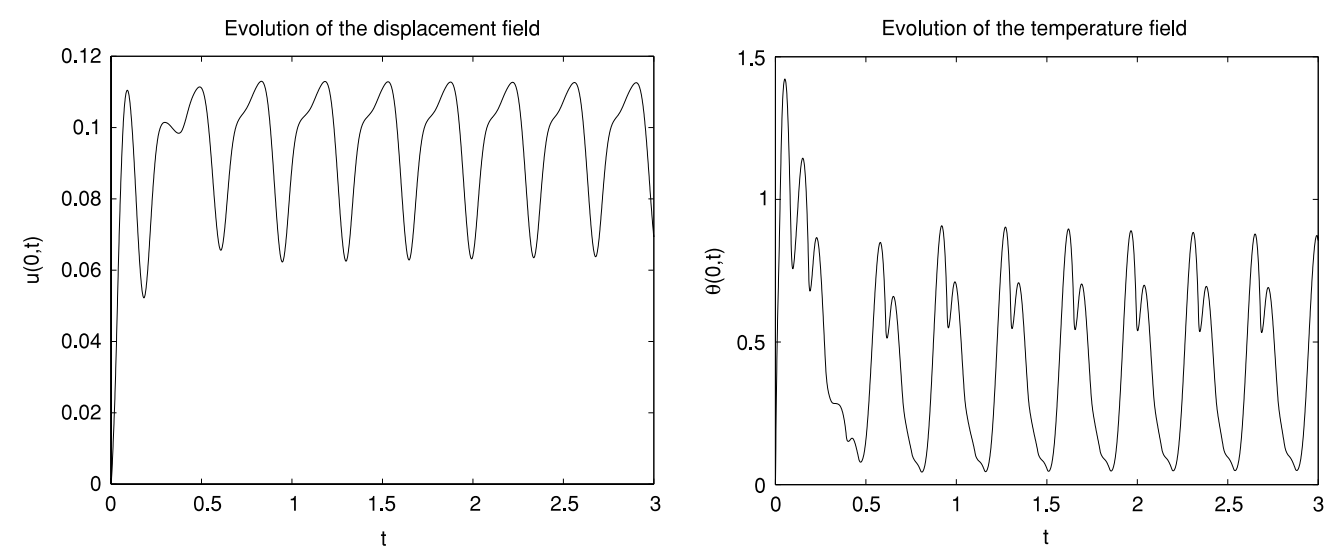

FIGURE 3. Example 1: Evolution of the displacements and the temperature at the contact point $x=0$.

which is implemented following the ideas of Fernández et al. [9], and using the calculations found in Viaño et al. [34]. Substituting $v_{n}^{h k}$ into (4.2) we find $\omega_{n}^{h k}$, and $\xi_{n}^{h k}$ is calculated from (4.3). We use these values in the linear system (4.4), which is solved to obtain the discrete beam temperature $\theta_{n}^{h k}$. Similarly, using $v_{n}^{h k}$ and $\omega_{n}^{h k}$ in (4.5), the discrete beam temperature $\theta_{w, n}^{h k}$ is calculated. Finally, we find $p_{n}^{h k}$ and $u_{n}^{h k}$ by using (4.6) and (4.7).

\section{Numerical results}

The FEM scheme described in $\S 4$ was implemented and the results of four numerical simulations are presented. In the examples the beam's length was $1 \mathrm{~m}(L=1)$ and the spatial discretization was given by $V^{h}, E^{h}$ and $B^{h}$. We used values $h=k=0.001$ as the discretization parameters.

\subsection{Example 1: Slow wheel and large applied pressure}

We consider a rotating wheel with an initial small angular velocity, $\omega_{0}=10$, while the applied pressure $p_{*}(t)$ is assumed to be constant and large $\left(p_{*}(t)=p_{*}=100\right)$. The beam starts from rest and its behaviour during 3 seconds (i.e. $T=3$ ) is computed. No volume forces act on it, and its deflection is caused by the contact with the rotating wheel.

The following data have been used:

$$
\begin{aligned}
& T=1, \quad L=1, \quad c^{2}=100, \quad d=0.001, \\
& k_{B}=10, \quad k_{w}=0.001, \quad \alpha=10, \quad h=0.1, \quad J=1, \\
& \gamma=0.5, \quad \delta_{w}=0.01, \quad \lambda_{1}=0.4, \quad \lambda_{2}=0.1, \quad \lambda_{3}=1, \\
& u_{0}=0, \quad v_{0}=0, \quad \theta_{0}=0, \quad \omega_{0}=10, \quad \xi_{0}=0 .
\end{aligned}
$$

In Figure 3 the evolution of the displacement at the contact point $x=0$ is shown on the left, and on the right the evolution of the temperature field. The oscillations of the beam's tip are clearly seen, superimposed on its deflection due to the rotation of the wheel. Also, the tip temperature oscillates as the frictional heat generated oscillates with its motion. 

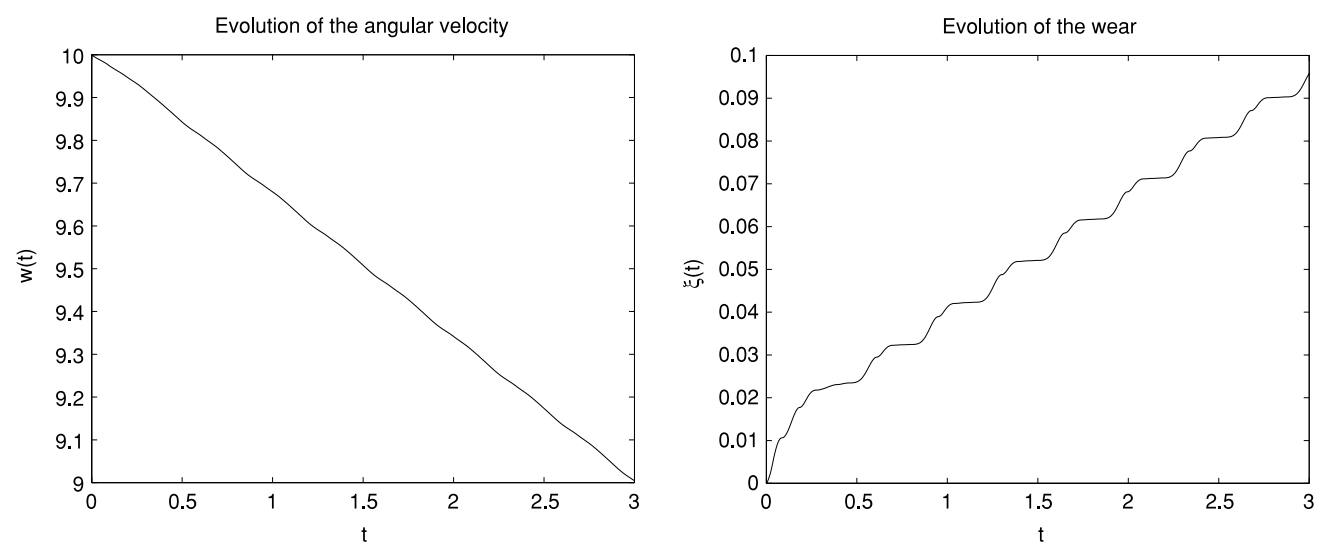

FIGURE 4. Example 1: Evolution of the wheel's angular velocity and of the wear.

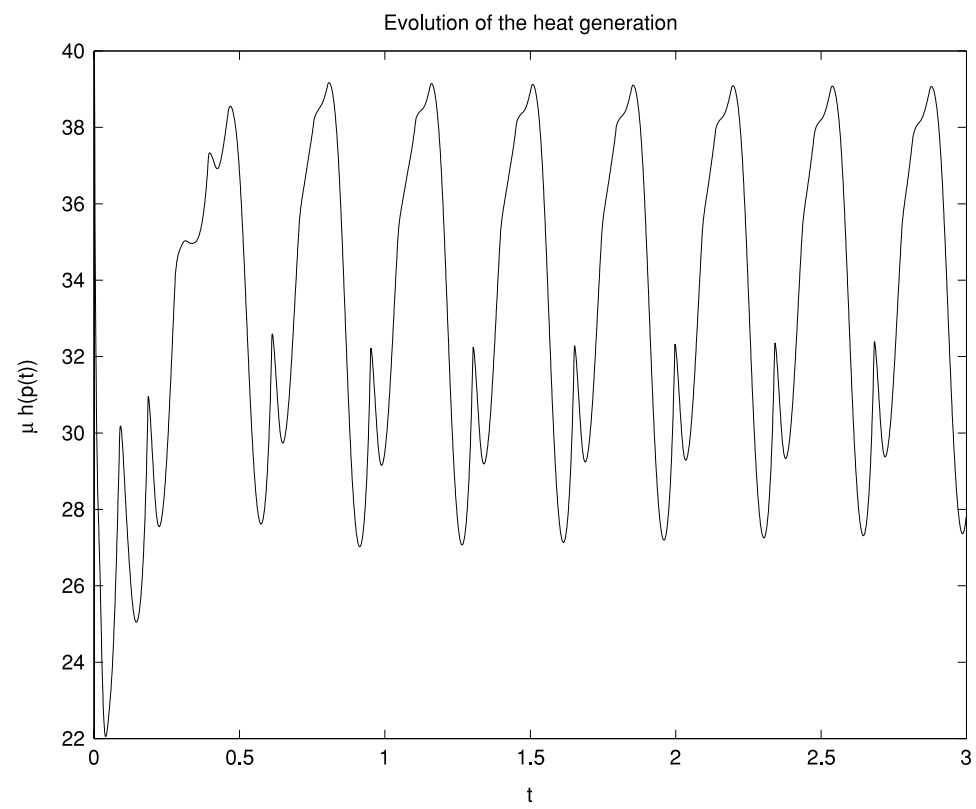

FIGURE 5. Example 1: Evolution of the heat generated.

In Figure 4 the evolution of the angular velocity of the wheel and the wear, due to friction, of the tip are depicted. It is seen that the angular velocity decreases almost linearly, but the wear, although increasing as was to be expected, is rapid when the contact pressure is high and slower when the pressure is lower. In Figure 5 the power lost as frictional heat is plotted. It correlates well with the oscillations in the tip temperature.

\subsection{Example 2: Fast wheel and medium applied pressure}

The setting is as in Example 1, except that now the initial angular velocity is assumed to be $\omega_{0}=100$ and the applied pressure $p_{*}=10$. 

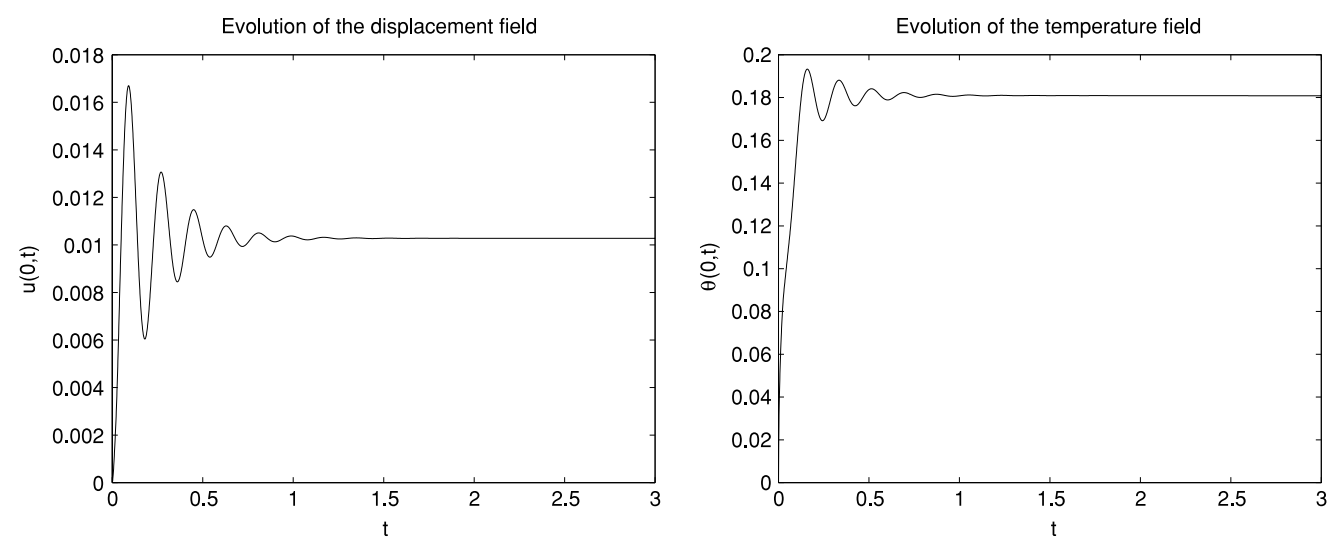

FIGURE 6. Example 2: Evolution of the displacements and temperature at $x=0$.
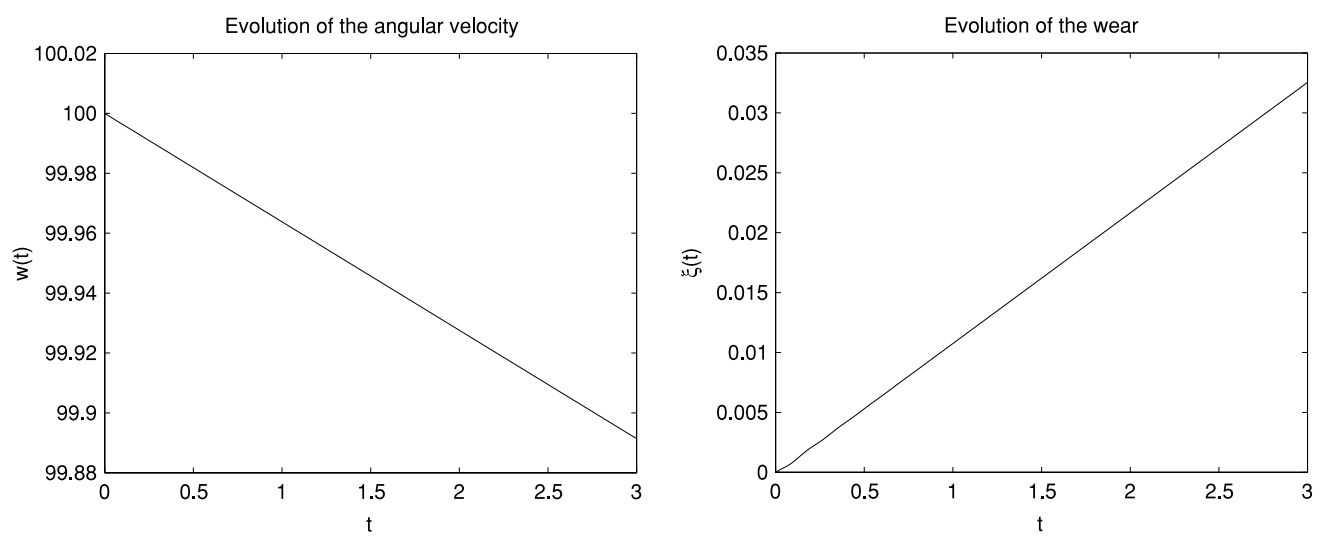

FIGURE 7. Example 2: Evolution of the angular velocity of the wheel and of the wear.

In Figure 6 the evolution of the displacement field at the contact point $x=0$ is shown on the left, and on the right that of the temperature field. The tip reaches a 'steady' position on the wheel rather quickly, and stays there for a while, since the wheel slows down very slowly, as can be seen in Figure 7 (left), where the angular velocity $v s$. time is depicted. The temperature of the tip settles down quickly, too.

The evolution of the wear is depicted in Figure 7 (right). In Figure 8 the frictional heat generated vs. time is plotted.

\subsection{Example 3: Stopping time of the wheel vs. applied pressure}

The main interest in this work is in the braking process, so we describe the stopping time $T^{*}$ as a function of the applied pressure $p_{*}$. The data is the same as in Example 1, except for $h=0.1$, which was chosen so as to reduce the CPU time. The time $T^{*}$ was that at which $\omega$ reached the value 0.001 . We plot it in Figure 9, and observe that the relationship is nonlinear, and there is a 'window' where a small increase in the applied pressure causes large difference in the stopping time. 


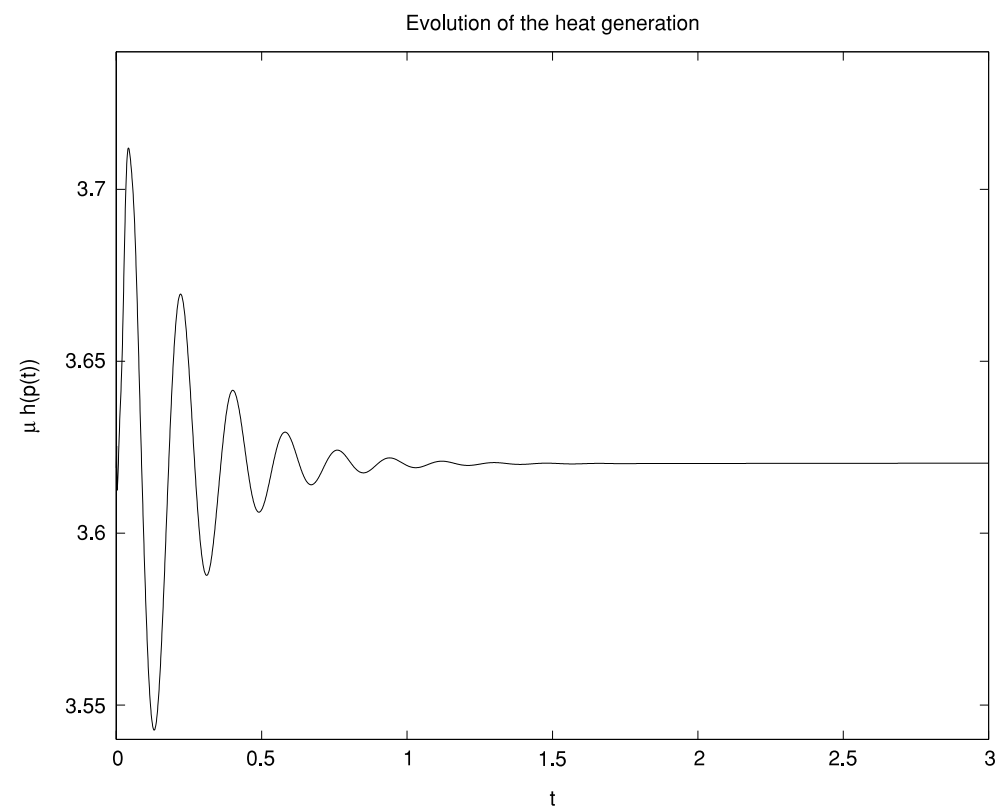

FIGURE 8. Example 2: Evolution of the generated heat.

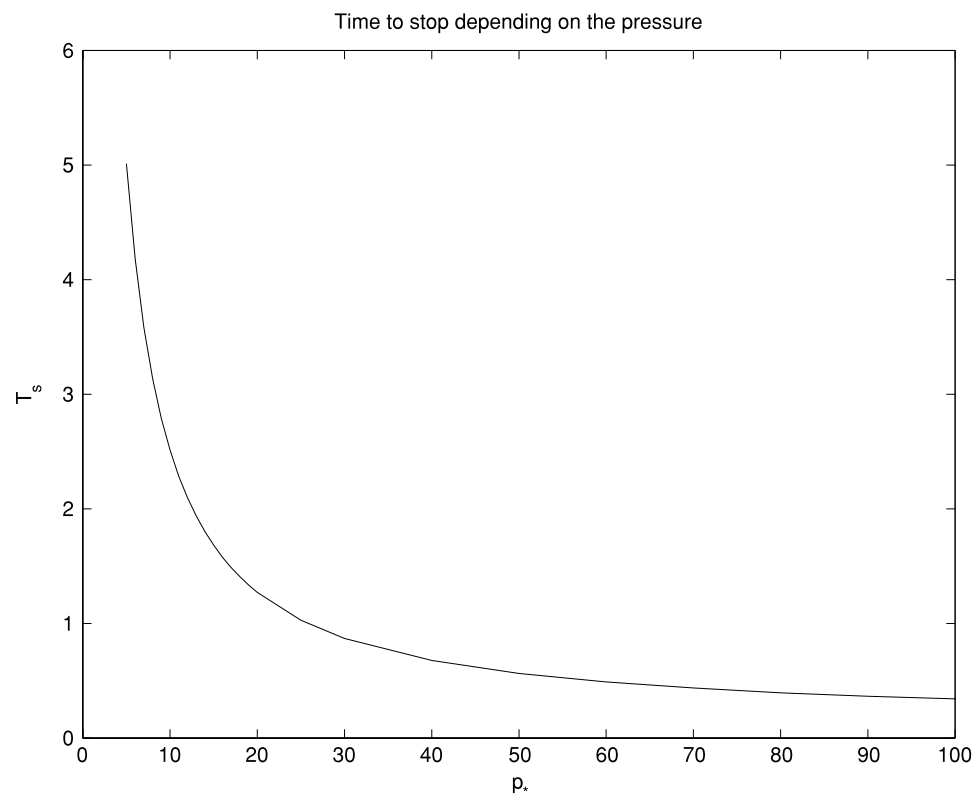

FigurE 9. Example 3: Time to stop $v$ s. the applied pressure.

\subsection{Example 4: Stopping time of the wheel vs. initial angular velocity}

In the last example we study the time needed to stop, $T^{*}$, as a function of the initial angular velocity of the wheel. The same data that in the previous example have been 


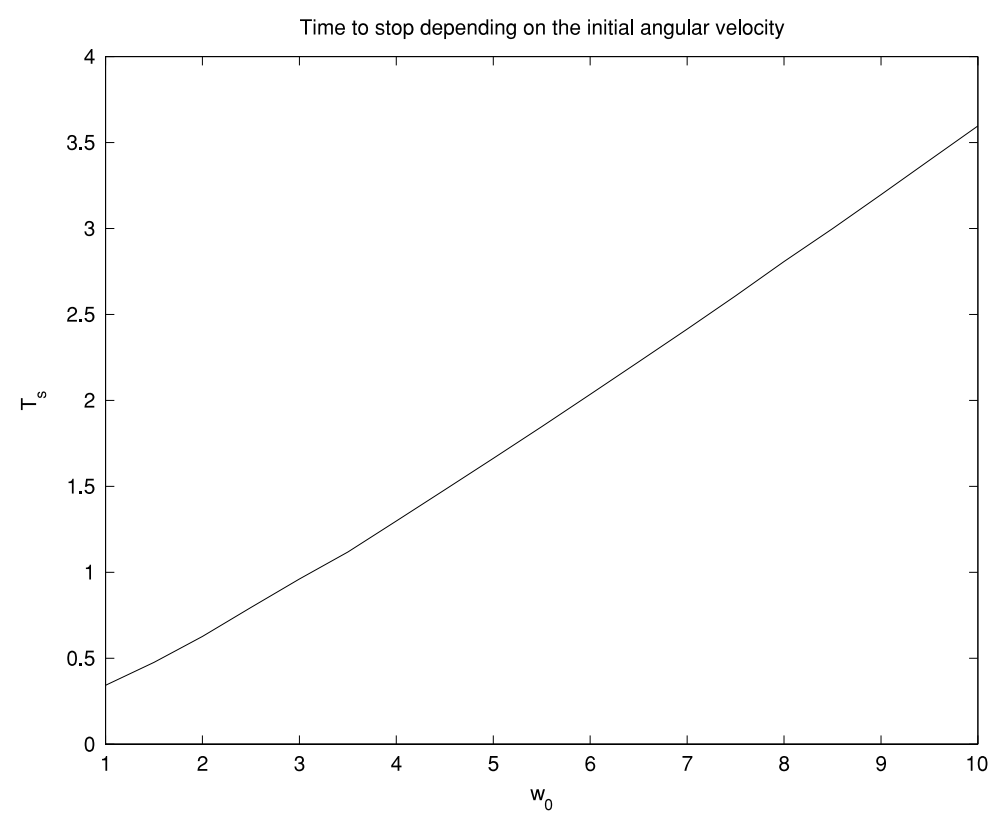

Figure 10. Example 4: Time to stop vs. initial angular velocity.

used. The time to stop is shown in Figure 10, and it is seen that it is almost linear, as a function of the initial angular velocity $\omega_{0}$.

\section{Conclusions}

A model for the braking of a rotating wheel, resulting from contact with a beam, was constructed. It consists of a coupled system of equations and inclusions for the beam displacements and temperature, the wheel temperature and angular velocity, and for the wear of the contacting tip. A variational formulation was obtained, and an existence and uniqueness results quoted from [23]. Further regularity of the solutions remains an open and important problem.

The interest in this work lies in the thermomechanical behaviour of the system, with emphasis on processes involved in contact. Friction was modelled with a slip-rate and temperature dependent friction coefficient, and frictional heat generation was included. Also, the wear of the contacting tip was taken into account. We note that the motion of the contacting tip is a part of the problem, and it led to the introduction of the free patch problem in Kuttler \& Shillor [22].

A FEM algorithm for the model was developed and implemented, and results of the numerical simulations were presented. The vibrations of the beam and of its tip lead to oscillations of the contact pressure and the rate of heat generation. In the examples, a slow wheel and large applied pressure and a fast wheel and a medium pressure were depicted. Whereas the stopping time was found to be linear as a function ot the initial angular velocity, it was found not linear as a function of the applied pressure. It was found, as can be seen in Figure 9, that large values of the applied pressure do not necessarily lead 
to the decrease of the stopping time. Moreover, the wear of the tip was increasing but with variable rate in Example 1.

This work is the first step in a program in which the contact parameters, in particular the dependence of the friction coefficient on the slip-rate and on temperature, and the wear condition, will be identified from experimental measurements, via parameter identification optimization approach. The setting is easier to set-up and much easier to perform measurements near the contacting patch. Also, it is easier to simulate numerically.

\section{References}

[1] Amassad, A., Shillor, M. \& Sofonea, M. (1999) A quasistatic contact problem with slip dependent coefficient of friction. Math. Meth. Appl. Sci. 22, 267-284.

[2] Andrews, K. T., Shi, P., Shillor, M. \& Wright, S. (1993) Thermoelastic contact with Barber's heat exchange condition. Appl. Math. Optim. 28, 11-48.

[3] Andrews, K. T., Kuttler, K. L. \& Shillor, M. (1997) On the dynamic behaviour of a thermoviscoelastic body in frictional contact with a rigid obstacle. Euro. J. Appl. Math. 8, $417-436$.

[4] Andrews, K. T., Klarbring, A., Shillor, M. \& Wright, S. (1997) On the dynamic behavior of a thermoviscoelastic contact problem with friction and wear. Int. J. Eng. Sci. 35(14), 1291-1309.

[5] Archard, J. F. (1953) Contact and rubbing of flat surfaces. J. Appl. Phys. 24(8), 981-988.

[6] Barber, J., Dundurs, J. \& Comninou, M. (1980) Stability considerations in thermoelastic contact. J. Appl. Mech. 47, 871-874.

[7] Duvaut, G. \& Lions, J. L. (1976) Inequalities in Mechanics and Physics. Springer.

[8] ECK, C. \& JARUSEK, J. (1999) The solvability of a coupled thermo-viscoelastic contact problem with small Coulomb friction and linearized growth of frictional heat. Math. Meth. Appl. Sci. 22, 1221-1234.

[9] Fernández, J. R., Shillor, M. \& Sofonea, M. (2002) Numerical analysis and simulations of quasistatic frictional wear of a beam. Preprint.

[10] Gosh, D., Basu, H. \& Manna, I. (1999) Mathematical modeling of thermal profile generated in the sample during a pin-on-disc wear testing operation. Scripta Materiala, 40(4), 417-423.

[11] Gu, R. J., Kuttler, K. L. \& Shillor, M. (2000) Frictional wear of a thermoelastic beam. J. Math. Anal. Appl. 242, 212-236.

[12] Gu, R. J. \& Shillor, M. (2001) Thermal and wear analysis of an elastic beam in sliding contact. Intl. J. Solids Structures, 38(14), 2323-2333.

[13] Han, W. \& Sofonea, M. (2002) Quasistatic Contact Problems in Viscoelasticity and Viscoplasticity. AMS and International Press.

[14] Han, W., Kuttler, K. L., Shillor, M. \& Sofonea, M. (2002) Elastic beam in adhesive contact. Int. J. Solids Structures, 39(5), 1145-1164.

[15] Ionescu, I. R. \& Paumier, J.-C. (1994) On the contact problem with slip dependent friction in elastodynamics. Euro. J. Mech. A/Solids, 13(4), 555-568.

[16] JANG, Y. H. (1999) Transient thermoelastic contact problems for an elastic foundation. Int. J. Solids Structures, 37, 1997-2004.

[17] Kinderlehrer, D. \& Stampacchia, G. (2000) An Introduction to Variational Inequalities and their Applications, 2nd ed. SIAM.

[18] Kuttler, K. L. (1986) Time dependent implicit evolution equations. Nonlinear Anal. 10(5), 447-463.

[19] Kuttler, K. L. \& Shillor, M. (1999) Set-valued pseudomonotone maps and degenerate evolution inclusions. Commun. Contemp. Math. 1(1), 87-123.

[20] Kuttler, K. L. \& Shillor, M. (2001) Dynamic bilateral contact with discontinuous friction coefficient. Nonlinear Anal. 45, 309-327. 
[21] Kuttler, K. L. \& Shillor, M. (2002) Dynamic contact with normal compliance wear and discontinuous friction coefficient. SIAM J. Math. Anal. 34(1), 1-27.

[22] Kuttler, K. L. \& Shillor, M. Heat conduction with flux condition on a free patch. Appl. Math. Optim. (to appear).

[23] Kuttler, K. L., Shillor, M. \& Fernández, J. R. (2002) Existence for a thermoviscoelastic beam model of brakes. Preprint.

[24] Lions, J. L. (1969) Quelques Methods de Resolution des Problemes aux Limites Non Lineaires. Dunod, Paris.

[25] Martins, J. A. C. \& Monteiro Marques, M. D. P. (eds.) (2002) Contact mechanics. Proc. 3rd CMIS Conference, Pehiche, Portugal. Kluwer.

[26] Osinski, Z. (ED.) (1998) Damping of Vibrations. Balkema, Rotterdam.

[27] Rabinowicz, E. (1958) The intrinsic variables affecting the stick-slip process. Proc. Roy. Phys. Soc. 71, 668-675.

[28] Rabinowicz, E. (1995) Friction and Wear of Materials (2nd ed). Wiley.

[29] Rochdi, M. \& Shillor, M. (2000) Existence and uniqueness for a quasistatic frictional bilateral contact problem in thermoviscoelasticity. Quart. Appl. Math. 38(3), 409-422.

[30] Shillor, M. (ED.) (2000) Recent advances in contact mechanics. Math. Comput. Model 28, (4-8).

[31] Shillor, M., Sofonea, M. \& Telega, J. J. Models and Analysis of Quasistatic Contact. In preparation.

[32] Strömberg, N., Johansson, L. \& Klarbring, A. (1996) Derivation and analysis of a generalized standard model for contact friction and wear. Int. J. Solids Structures, 13, 1817-1836.

[33] Telega, J. J. (1998) Topics on unilateral contact problems of elasticity and inelasticity. In: Moreau, J. J. and Panagiotoupolos, P. D. (eds.), Nonsmooth Mechanics and Applications, pp. 340-361. Springer.

[34] Viaño, J. M. \& Figueiredo, J. (n.d.) Implementaão do método de elementos finitos, Curso de Mestrado em Matemática e Aplicaões à Mecânica. Publications of Departamento de Matemática da Universidade do Minho.

[35] Yevtushenko, A. A. \& Ukhanska, O. M. (1993) Plane contact problem of thermoelasticity with quasi-steady frictional heating. Int. J. Eng. Sci. 31, 1565-1573.

[36] Yi, Y.-B., Barber, J. R. \& HARTSOCK, D. L. (2001) Thermoelastic instabilities in automotive disk brakes - Finite element analysis and experimental verification. In: Martins, J. A. C. and Monteiro Marques, M. D. P. (eds.), Contact Mechanics, Pehiche, Portugal. Kluwer.

[37] Zmitrowicz, A. Variational approach to contact, friction and wear problems. ZAMM (to appear). 